\title{
Magnetic Fluctuations and Peculiar Anisotropy Oscillations in the 23 July 2012 SEP Event
}

\author{
R. A. Leske ${ }^{\star a}$, A. C. Cummings ${ }^{a}$, C. M. S. Cohen ${ }^{a}$, R. A. Mewaldt ${ }^{a}$, A. W. Labrador ${ }^{a}$, \\ E. C. Stone ${ }^{a}$, M. E. Wiedenbeck ${ }^{b}$, E. R. Christian ${ }^{c}$, and T. T. von Rosenvinge ${ }^{c}$ \\ ${ }^{a}$ California Institute of Technology, Pasadena, CA 91125 USA \\ ${ }^{b}$ Jet Propulsion Laboratory, California Institute of Technology, Pasadena, CA 91109 USA \\ ${ }^{c}$ NASA/Goddard Space Flight Center, Greenbelt, MD 20771 USA \\ E-mail: ralesrl.caltech.edu, ace@srl.caltech.edu, \\ cohendsrl.caltech.edu, rmewaldtesrl.caltech.edu, \\ labradorasrl.caltech.edu, ecsesrl.caltech.edu, \\ Mark.E.Wiedenbeck@jpl.nasa.gov, Eric.R. Christian@nasa.gov, \\ tycho.t.vonrosenvinge@nasa.gov
}

\begin{abstract}
The pitch-angle distributions of solar energetic particles (SEPs) provide a wealth of information about particle transport through interplanetary space, and depend on the magnetic field strength, topology, and turbulence at remote heliospheric locations. The Low Energy Telescope (LET) on STEREO measures angular distributions in the ecliptic for SEP ions from protons to iron with energies of about 2-12 MeV/nucleon. At the onset of the 23 July 2012 extreme SEP event, a beamed distribution was observed at STEREO-Ahead using LET. We have previously reported apparent "oscillations" in the pitch-angle width of this beam when using simultaneous particle and magnetic field measurements at a 1-minute cadence. We have now determined that this behavior is largely a result of the effects of magnetic turbulence on the calculated particle pitch angle distributions. The locally measured magnetic field direction varies relatively rapidly, while the energetic particle distribution is governed by magnetic fields averaged over much larger spatial and temporal scales than represented by solar wind measurements at the same cadence. Similar behavior in earlier events has been reported by others and analyzed in the framework of quasilinear theory.
\end{abstract}

35th International Cosmic Ray Conference - ICRC2017

10-20 July, 2017

Bexco, Busan, Korea

* Speaker. 


\section{Introduction}

An extreme solar energetic particle (SEP) event was detected at the Ahead spacecraft of the Solar TErrestrial RElations Observatory (STEREO) [1] pair on 23 July 2012 originating from an active region over the limb from Earth at $\sim \mathrm{W} 133^{\circ}$, or near central meridian from the vantage point of STEREO-Ahead at the time [2, 3, 4]. The event was associated with an x-ray flare estimated to be $\sim X$ class [5] and included a blast wave observed in situ at Ahead at 20:55 UT with speeds $>2000 \mathrm{~km} / \mathrm{s}$ and interplanetary magnetic field strengths eventually reaching over $100 \mathrm{nT}$ [2].

The Low Energy Telescope (LET) onboard STEREO measures SEP anisotropies in 16 different in-ecliptic viewing directions with angular bins $\geq 12.5^{\circ}$ wide for 10 different element and energy bands from $\mathrm{H}$ through $\mathrm{Fe}$ at about $2-12 \mathrm{MeV} /$ nucleon [6]. The instrument field of view is limited to about $\pm 15-20^{\circ}$ of latitude but encompasses two back-to-back fans each spanning $133^{\circ}$ of longitude centered along the nominal Parker spiral field direction, leaving a pair of $47^{\circ}$-wide longitudinal gaps perpendicular to the nominal magnetic field that cannot be observed.

In previous reports, we have discussed LET/Ahead anisotropy observations in the 23 July 2012 event $[7,8]$. In particular, we noted puzzling oscillations in the width of a beamed distribution at the onset of this event and speculated as to their cause. As described below, we have now determined that much of the apparent oscillatory behavior is illusory, and results from calculating pitch angles relative to a magnetic field that is not aligned with the symmetry axis of the particle distribution. Turbulent fluctuations in the field direction on scales small compared to the gyroradius of the energetic particles introduce distortions into the calculated pitch angle distributions.

\section{Observations and Analysis}

Shown in the middle panel of Figure 1 are time intensity profiles at the onset of the 23 July 2012 event from LET on Ahead in each of 16 bins in $\mu$ (where $\mu$ is the cosine of the pitch angle) at 1-minute time resolution. In both the outward-flowing ( $\mu=0.94)$ and inward-flowing $(\mu=-0.94)$ field-aligned directions the intensities rose rather smoothly. However, for intermediate values of $\mu$ in the forward hemisphere $(\mu>0)$ that are not field-aligned, time profiles were not smooth and intensities varied tremendously, by factors of 5 or so on timescales of $\sim 10-15$ minutes. Throughout this period, the magnetic field direction was mostly within the LET field of view (Figure 2 of [7]). The peculiar shapes and rapid variability of the pitch-angle distributions (PADs) are more easily seen in the selected 1-minute samples in the bottom panel of Figure 1; at times the beam width fluctuated repeatedly by $>25^{\circ}$ in a matter of minutes, and the distributions often tended to flatten at the peaks. In our earlier reports $[7,8]$ we had failed to notice that changes in the variability of the PADs are well-correlated with changes in the variability of the magnetic field direction. Note that in the top panel of Figure 1 the magnetic field longitude varied over a range of $>50^{\circ}$ between $\sim 5: 30$ and 6:10 UT (sometimes varying by $20^{\circ}$ or more from one minute to the next within this interval), exactly when the time intensity profiles in many $\mu$ bins (middle panel) were most variable. Conversely, when the magnetic field longitude became much more steady after $\sim 7: 10$ UT, the intensity variations were greatly reduced.

If PADs are calculated relative to the "wrong" magnetic field directions (i.e., directions not aligned with the particle distribution symmetry axis), as illustrated in Figure 2, this results in broad- 

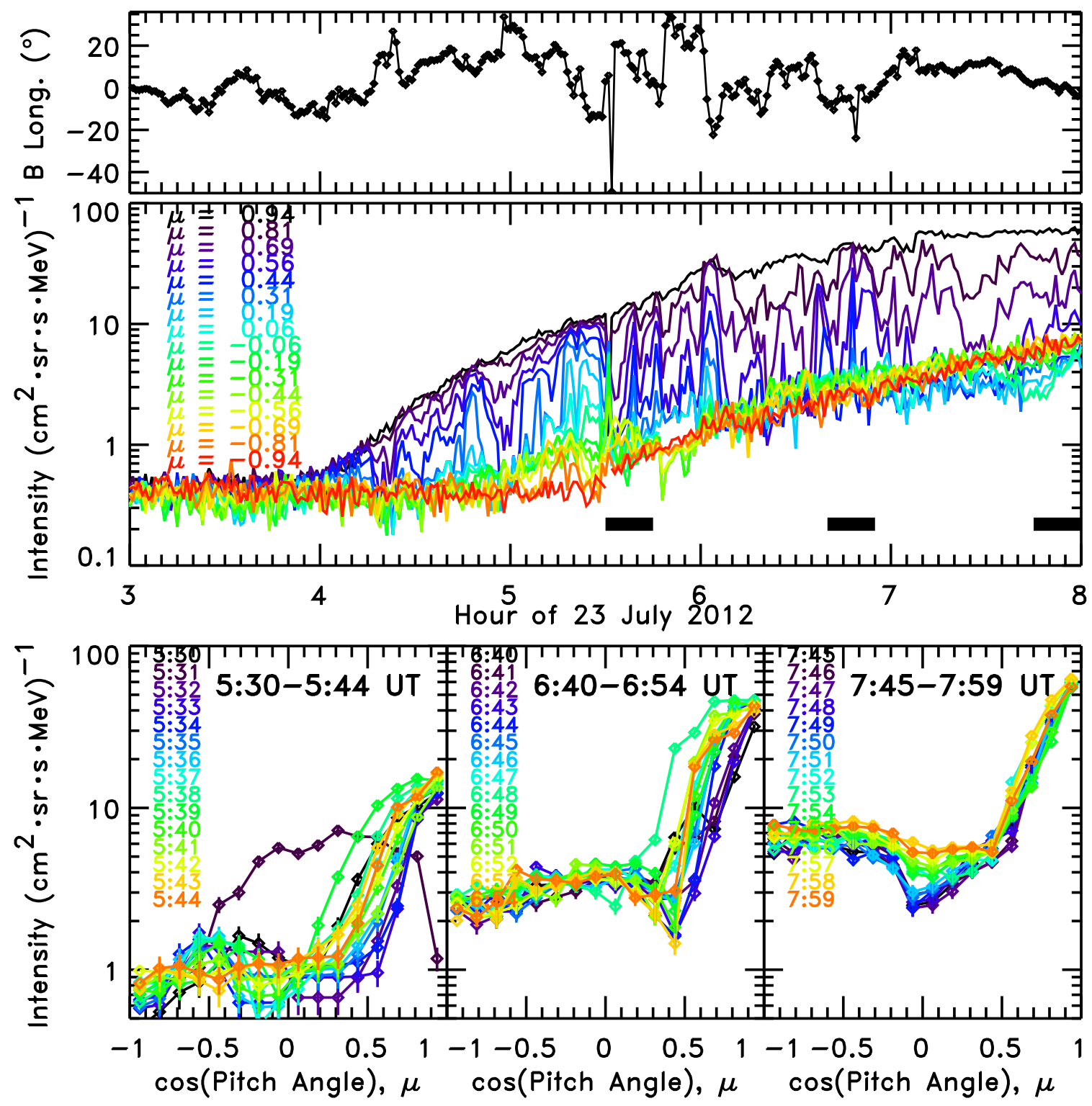

Figure 1: Top: One-minute averaged magnetic field longitude (relative to the Sun at $0^{\circ}$ ) from MAG on STEREO-Ahead at the onset of the 23 July 2012 SEP event. Middle: Time profiles at 1-minute resolution for 6-10 MeV sectored $\mathrm{H}$ in each of 16 pitch-angle bins from LET on STEREO-Ahead. Bottom: Sample one-minute averaged pitch-angle distributions for 6-10 MeV H in LET at STEREO-Ahead on 23 July 2012 at the times indicated, corresponding to the black bars in middle panel.

ened, flattened PADs that are more variable in width when the field direction is more variable, much as we observe (Figure 1). For the calculations represented in Figure 2, the standard assumption of gyrotropy was employed, that is, if multiple intensity measurements exist at the same angular separation from the field but in different clock angle directions, simply use the average of the measurements. (Of course, real measurements as in Figure 4 often have sizable uncertainties and are spaced more sparsely in longitude than the idealized curves in Figure 2.)

We have investigated the possibility of a time offset between the MAG and LET datasets used 

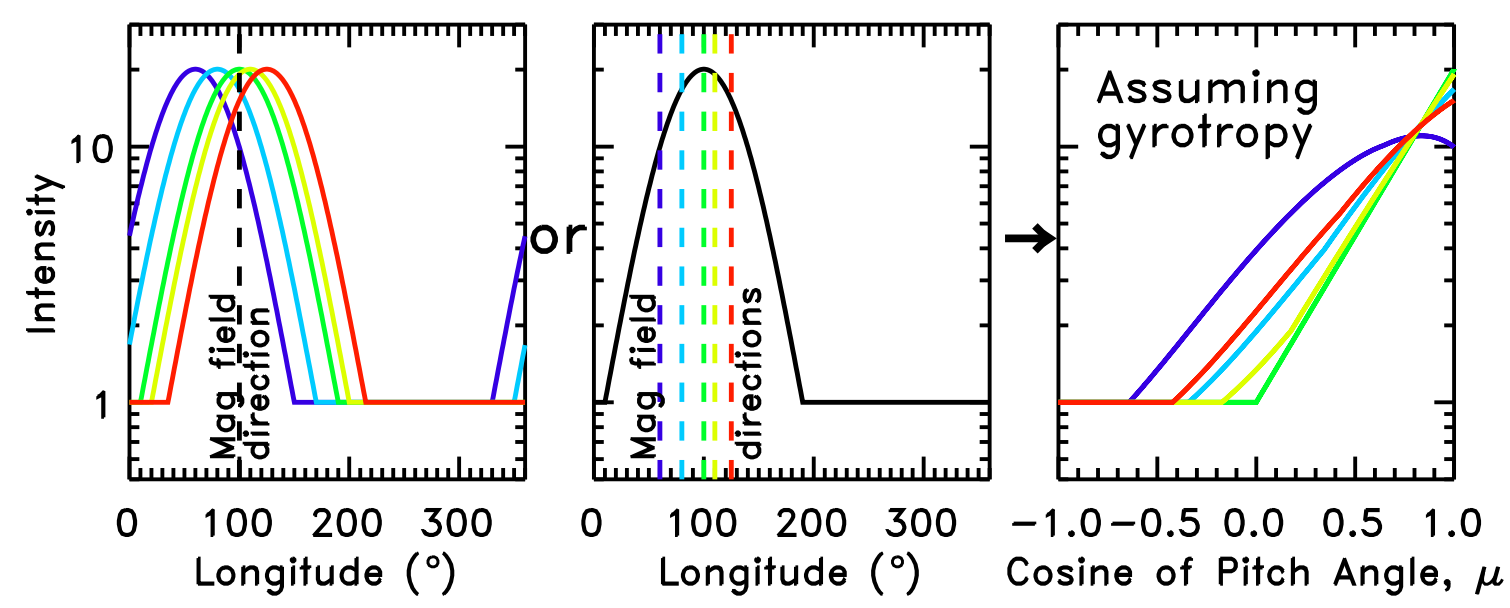

Figure 2: Schematic illustration of how identical longitudinal angular distributions of particles will yield varying, distorted pitch angle distributions (PADs) if the PADs are calculated relative to field directions not aligned with the distribution symmetry axis and gyrotropy is assumed. Color in either of the first two panels matches that of the resulting curve in the third panel.

for our pitch angle calculations, which would produce mismatches between the field directions and particle distribution peak directions when either is varying rapidly. Onboard the STEREO spacecraft, data collected by LET during one minute are transferred to the data processing unit (DPU) during the following minute. The DPU then assigns the time tag using the time of the preceeding minute, so that the time telemetered with the data correctly refers to the beginning of the minute in which the data were actually accumulated [9]. However, initially we were unaware that in publicly available MAG data at http://www-ssc.igpp.ucla.edu/forms/stereo/level2_plasma_and_magnetic_ field.html, any time tag listed refers to the midpoint of the data accumulation interval [C. Russell, private communication], while for LET data at http://www.srl.caltech.edu/STEREO/Public/LET_ public.html time stamps mark the beginning of the averaging interval as stated in the data files. Furthermore, we had neglected the fact that LET 1-minute data are not aligned on minute boundaries, but rather have an offset varying throughout the mission as the instrument clock drifts slightly; for the period of interest here, this offset amounts to 58 seconds. Thus, in our previous reports $[7,8]$ we were inadvertently comparing LET data with magnetic field directions measured 88 seconds earlier. To correct this error, we obtained higher cadence $(8 \mathrm{~Hz})$ magnetic field data and created minute averages using the time boundaries in the LET data for use in calculating the PADs shown here in Figure 1. Only small differences can be seen when comparing with Figures 4 and 5 in [7]; clearly the "oscillations" persist here and therefore they are not solely (or even primarily) due to a simple time offset between the two datasets.

Instead, we believe the root cause of the oscillatory behavior lies with the vastly different spatial and temporal scales sampled by the energetic particles compared with the solar wind that carries the magnetic field. The geometric mean energy of the $6-10 \mathrm{MeV}$ protons used in Figure 1 is $7.75 \mathrm{MeV}$, corresponding to a velocity of $0.13 c$, or $\sim 88$ times faster than that of the solar wind (which was $430 \mathrm{~km} / \mathrm{s}$ at the event onset). In the $\sim 4 \mathrm{nT}$ magnetic field prevalent during this time, the proton gyroperiod is $\sim 17 \mathrm{~s}$. Thus, it takes the solar wind $\sim 25$ minutes to cover the same distance traversed by field aligned $6-10 \mathrm{MeV}$ protons during a single gyroperiod, and the magnetic field 

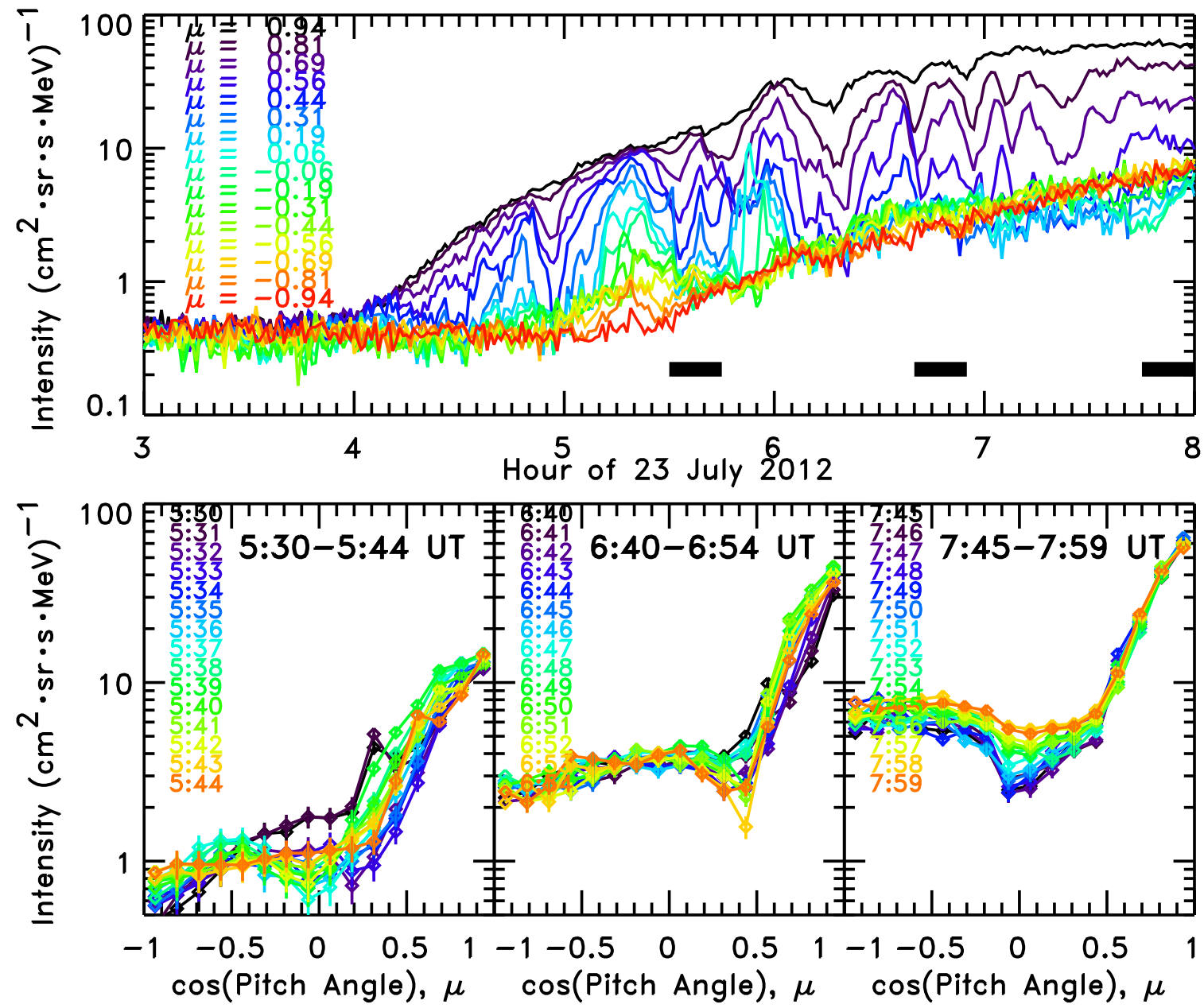

Figure 3: Same as the bottom two panels of Figure 1, except that here pitch angles were calculated relative to field directions obtained by averaging for up to 25 minutes (see text), rather than directions measured simultaneously with the particles.

direction fluctuates tremendously during any 25 minute interval between $\sim$ 04:00 and $\sim$ 07:00 UT (top panel of Figure 1). The trajectories of these protons and their angular distribution are governed by an average magnetic field whose direction may be quite different from that instantaneously measured when the protons arrive at LET.

Calculating the direction of the mean field guiding the particles is impossible without detailed knowledge of the magnetic field in a large region around the spacecraft (the proton gyroradius under the above conditions is $\sim 100,000 \mathrm{~km}$ ), which is unavailable from a single-point measurement (although models of particle transport in a turbulent field may provide some general insight). However, as a test of this idea we show in Figure 3 the results of recalculating the pitch angles in Figure 1 relative to field directions averaged by up to 25 minutes. Specifically, for particles arriving from the Sunward direction, we use the average of the measured field direction from the time of particle arrival, $t_{0}$, until $t_{0}+25$ minutes, while for those coming directly from the anti-Sun direction, the averaging period runs from $t_{0}-25$ minutes until $t_{0}$ (since the field these particles encountered has already passed the spacecraft). For particles arriving in viewing sectors with a boresight angle 


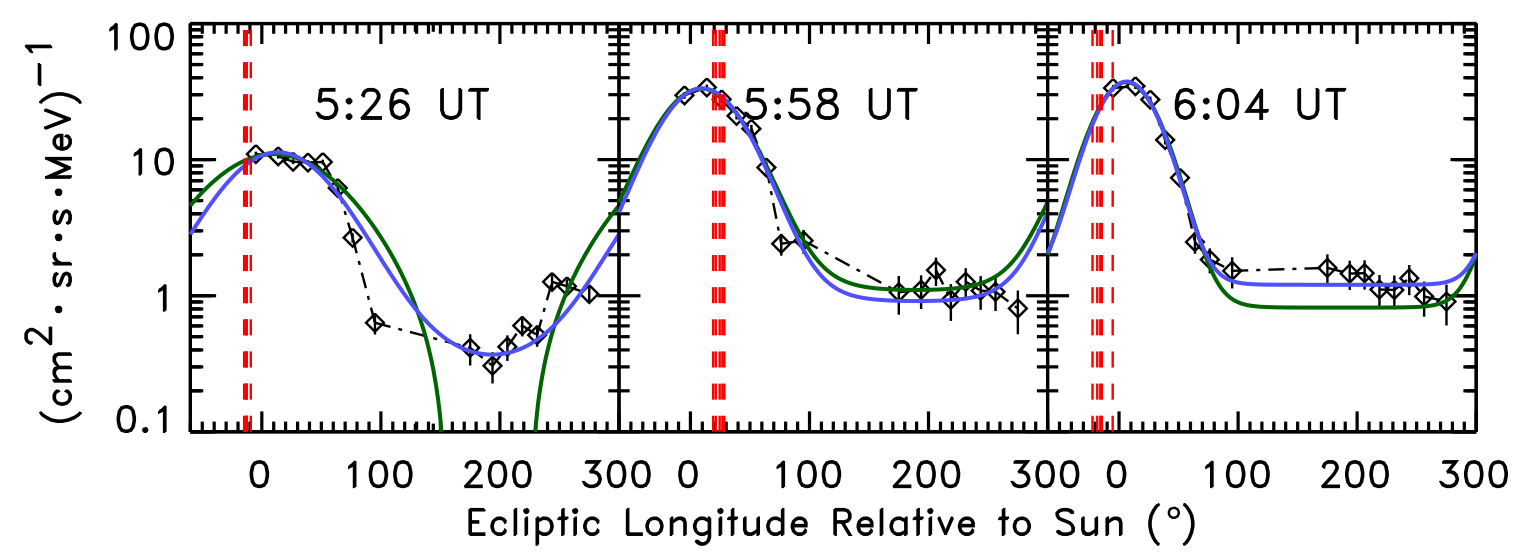

Figure 4: 6-10 MeV H one-minute sectored intensities (diamonds) from LET on STEREO-Ahead at the times indicated on 23 July 2012 vs. ecliptic longitude, compared with weighted (green) and unweighted (blue) fits to Equation 3.1. One-minute averaged magnetic field longitudes in a 5-minute window centered on each time are shown (vertical red lines) for comparison with the intensity peak directions.

$\phi$ off the Sun-spacecraft line, the average runs from $t_{0}$ out to $t_{0}+25 \cos \phi$ minutes. We have no expectation that this averaging scheme will accurately represent the true mean field guiding the particles, since (a) it is not clear that averaging over one full gyroperiod gives the correct direction, (b) knowledge of the field direction encountered by the particles arriving from off the (radial) solar wind flow direction is lacking, and (c) even along the radial direction, the field may well have evolved significantly over the \pm 25 minute interval from the measured value at the midpoint of that interval. Therefore we expect the "oscillations" will not be completely eliminated, but nevertheless we see they are very significantly reduced. The PADs in particular are much less variable and more sharply peaked along $\mu=1$ (compare bottom panels of Figures 1 and 3), and much of the remaining spread at a given $\mu$ is time-ordered and reflects the overall increase in particle intensity. Other averaging approaches using longer time windows or even a fixed field direction throughout this period show differences in the details of the resulting PADs, but all show a reduction in variability from the original, standard approach of Figure 1. We conclude that in general the average field direction is at least closer to that actually guiding the particles than the field direction instantaneously measured, since use of an arbitrary, unrelated field direction would only distort the resulting PADs (as shown in Figure 2).

\section{Discussion}

Our deduction that the particle distribution symmetry axes and instantaneous magnetic field directions often differ was rather indirect, based on the variable, distorted shapes of the resulting calculated PADs. We could directly determine the longitudinal angle of the peak of the particle distributions (LET is insensitive to latitude [6]) if we knew what function should fit the data. We have fit the measured sector intensities, $I$, as a function of ecliptic longitude, $\phi$, with the function

$$
I=A e^{B \cos \left(\phi-\phi_{0}\right)}+C,
$$

where the peak longitude is at $\phi_{0}$, and $A, B$, and $C$ are other fitted constants. This form was motivated by the expectation that the resulting PADs should be exponential in $\mu$, or cosine of 


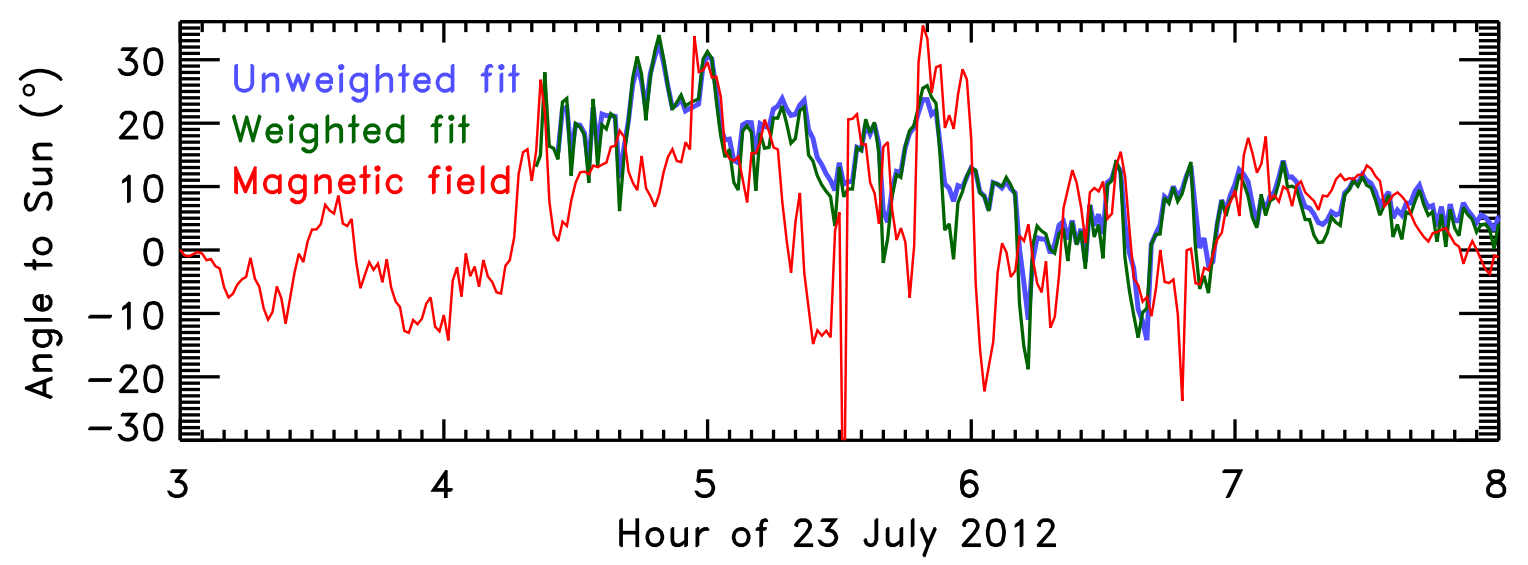

Figure 5: Magnetic field longitude (as in top panel of Figure 1) vs. time (red) compared with longitudes of the peaks in the particle angular distributions obtained from weighted (green) and unweighted (blue) fits to Equation 3.1.

the angle to the field. Even if this were true, Equation 3.1 would not provide a good fit if the symmetry axis is far out of the ecliptic and/or if intensities in the backward hemisphere are anything other than a constant (e.g., if the distributions were bidirectional). However, here we are only concerned with constraining the location of the peak, not the details of its overall shape. We note that, without the additive constant $C$, Equation 3.1 is a Gaussian for values of $\phi$ near $\phi_{0}$ (as is readily seen by replacing the cos function with its Taylor series expansion) and is known in the field of directional statistics as the von Mises distribution or the circular normal distribution (https://en.wikipedia.org/wiki/Directional_statistics).

In Figure 4 we show examples of fits to the 1-minute sectored data using Equation 3.1, for periods selected to have the magnetic field direction relatively steady for at least several minutes. The fact that in each case the field direction is different by up to tens of degrees from that of the peak of the particle distributions is therefore real and not due to remaining unrecognized time offsets between LET and MAG. The resulting fit peak locations over this entire period are shown in Figure 5, where the typical formal uncertainty at each point is about $\pm 2^{\circ}$. Both weighted and unweighted fits to the data were used. Weighted fits do better at fitting the points at the peak, but sometimes at the expense of points on the tail or opposite the peak (first panel of Figure 4). Given the close agreement between the two fits in Figure 5, the fitted peak location generally appears rather robust. There are clearly periods when the particle distribution is not centered on (and therefore, not gyrotropic about) the instantaneous, locally-measured field, and the particle and field directions are not simply time-shifted relative to each other.

Variations of the particle distribution symmetry axis from the local field direction similar to those presented here were reported 30 years ago by Bieber \& Evenson [10], although they did not discuss the shape of the PADs. These authors used a statistical treatment of the correlation between the magnetic field fluctuations and particle distribution fluctuations to infer details of the turbulent magnetic field structure probed by the particles in directions orthogonal to that of the solar wind flow. Their analysis was done in the framework of quasilinear theory and interpreted in terms of axisymmetric slab turbulence. In future work it would be interesting to apply their approach to 
analysis of our data in order to obtain a better understanding of turbulence in the extreme 23 July 2012 SEP event.

\section{Acknowledgments}

This work was supported by the National Aeronautics and Space Administration (NASA) at the California Institute of Technology and the Jet Propulsion Laboratory under sub-contract 00008864 from the University of California at Berkeley under NASA grant NNX15AG09G. We thank the STEREO/MAG team for making their data publicly available, and we thank Andrew Davis and Chris Russell for help in understanding the LET and MAG data timetags.

\section{References}

[1] M. L. Kaiser, T. A. Kucera, J. M. Davila, O. C. St. Cyr, M. Guhathakurta, and E. Christian, The STEREO Mission: An Introduction, Space Sci. Rev. 136 (2008) 5-16.

[2] C. T. Russell, R. A. Mewaldt, J. G. Luhmann, et al., The Very Unusual Interplanetary Coronal Mass Ejection of 2012 July 23: A Blast Wave Mediated by Solar Energetic Particles, ApJ 770 (2013) 38.

[3] R. A. Mewaldt, C. T. Russell, C. M. S. Cohen, et al., A $360^{\circ}$ View of Solar Energetic Particle Events, Including One Extreme Event, Proc. 33rd Internat. Cosmic Ray Conf. (Rio de Janeiro) (2013) paper 1186.

[4] Y. D. Liu, J. G. Luhmann, P. Kajdič, et al., Observations of an extreme storm in interplanetary space caused by successive coronal mass ejections, Nature Communications 5 (2014) 3481, [arXiv:1405.6088].

[5] N. V. Nitta, M. J. Aschwanden, P. F. Boerner, S. L. Freeland, J. R. Lemen, and J.-P. Wuelser, Soft $X$-ray Fluxes of Major Flares Far Behind the Limb as Estimated Using STEREO EUV Images, Solar Phys. (2013) [arXiv:1304.4163].

[6] R. A. Mewaldt, C. M. S. Cohen, W. R. Cook, et al., The Low-Energy Telescope (LET) and SEP Central Electronics for the STEREO Mission, Space Sci. Rev. 136 (2008) 285-362.

[7] R. A. Leske, A. C. Cummings, C. M. S. Cohen, et al., Insights Into Particle Transport Obtained from Solar Energetic Particle Anisotropies, in Proc. 34th Internat. Cosmic Ray Conf. (The Hague), PoS(ICRC15)072, (2015).

[8] R. A. Leske, A. C. Cummings, C. M. S. Cohen, et al., Solar Energetic Particle Anisotropies and Insights Into Particle Transport, in Solar Wind 14 (L. Wang, R. Bruno, E. Möbius, et al., eds.), vol. 1720 of AIP Conf. Proc., pp. 070004-1-070004-4, American Institute of Physics, 2016.

[9] B. Kecman, Low Energy Telescope Interface Control Document for the Solar Energetic Particle Central Electronics, Tech. Rep. STEREO-CIT-009.A, California Institute of Technology, 2003.

[10] J. W. Bieber and P. A. Evenson, Correlation of Variations in the Angular Distribution of $50 \mathrm{MeV}$ Particles with Magnetic Field Fluctuations, Proc. 20th Internat. Cosmic Ray Conf. (Moscow) 3 (1987) 151. 